\title{
Inverse cascade in the structure of substorm aurora and non-linear dynamics of field-aligned current filaments
}

\author{
B. V. Kozelov, I. V. Golovchanskaya, and O. V. Mingalev \\ Polar Geophysical Institute, Academgorodok, 26, Apatity, Murmansk region, 184209 Russia \\ Received: 24 June 2011 - Revised: 2 August 2011 - Accepted: 3 August 2011 - Published: 9 August 2011
}

\begin{abstract}
We investigate time evolution of scaling index $\alpha_{A}$ that characterizes auroral luminosity fluctuations at the beginning of substorm expansion. With the use of UVI images from the Polar satellite, it is shown that $\alpha_{A}$ typically varies from values less than unity to $\sim 1.5$, increasing with breakup progress. Similar scaling features were previously reported for fluctuations at smaller scales from all-sky TV observations. If this signature is interpreted in terms of non-linear interactions between scales, it means that the power of smallscale fluctuations is transferred with time to larger scales, a kind of the inverse cascade. Scaling behavior in the aurora during substorm activity is compared with that in the fieldaligned currents simulated numerically in the model of nonlinear interactions of Alfvénic coherent structures, according to the Chang et al. (2004) scenario. This scenario also suggests an inverse cascade, manifesting in clustering of smallscale field-aligned current filaments of the same polarity and formation of "coarse-grained" structures of field-aligned currents.
\end{abstract}

Keywords. Magnetospheric physics (Auroral phenomena; Magnetosphere-ionosphere interactions; General or miscellaneous)

\section{Introduction}

It has long been known that optical aurora both in quiescence and under disturbed conditions reveals signatures of scaleinvariance. This feature was first reported from visual inspection of auroral images (e.g. Oguti, 1975; Trondsen and Cogger, 1998). Later, scale-free statistics of the aurora was evidenced within frameworks of self-organized criticality and, alternatively, of intermittent turbulence that were invoked to

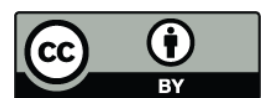

Correspondence to: B. V. Kozelov

(boris.kozelov@gmail.com) describe auroral dynamics as a manifestation of transients in the magnetosphere-ionosphere system (Uritsky et al., 2002; Kozelov, 2003; Kozelov et al., 2004; Kozelov and Rypdal, 2007; Klimas et al., 2010). In both approaches, power law distributions of various auroral characteristics over temporal and/or spatial scale, a signature of scale-independent regime, were stated.

A detailed study of turbulence manifestations in the aurora has been performed by Golovchanskaya et al. (2008), and practical guidelines for derivation of aurora scaling parameters from ground-based imaging observations were given by Kozelov and Golovchanskaya (2010).

The observed power-law distributions in the emission intensity over scales for substorm aurora indicate that scaling index $\alpha_{A}$ undergoes a characteristic temporal variation, increasing with development of auroral activity. This was previously reported by Kozelov and Rypdal (2007) by TV allsky observations. For larger spatial scales observed by the Polar UVI imager, a similar temporal variation of the roughness exponent of self-affine auroral surfaces was reported by Uritsky et al. (2006). This finding for larger scales is verified in the present study by examining Polar UVI images with an advanced wavelet technique (Abry et al., 2000).

We note that scaling properties in the aurora are often investigated ignoring their connection with electrodynamic processes in the magnetospheric plasma that most likely bring them to existence. It is well-known that, while the brightest aurora is usually associated with substorm events on the nightside, auroral structures are observed at all MLTs, forming the global-scale auroral oval (Feldstein and Starkov, 1967). Therefore the electrodynamic processes associated with the structured aurora can not be specific for substorm time only, but should have more common physical ground. Clearly, they should also manifest in the structured electric and magnetic fields, and it is very likely that the broadband extra low frequency (BB ELF) perturbations often observed in the auroral ionosphere at frequencies from tenths

Published by Copernicus Publications on behalf of the European Geosciences Union. 


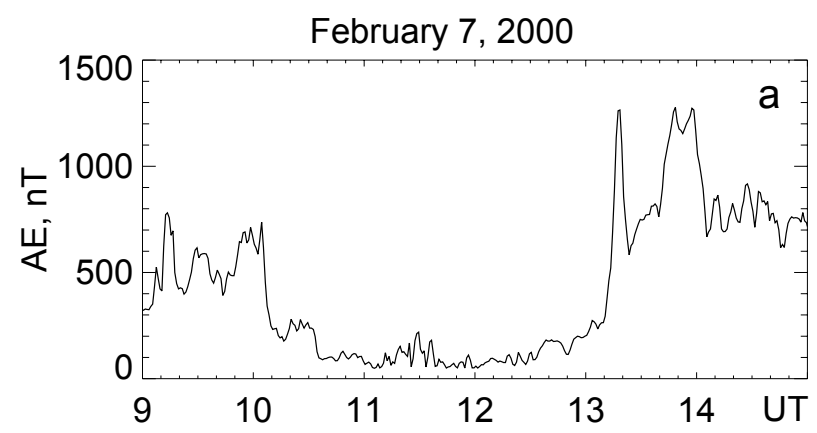

Feb 7, 2000,12:26:06

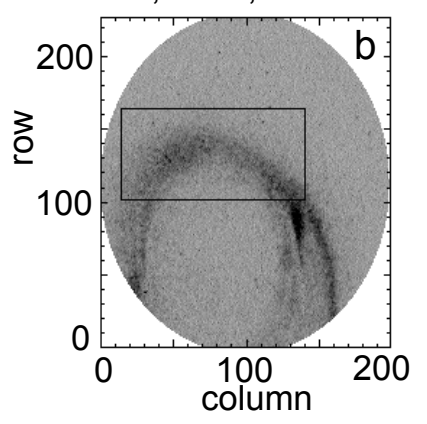

Feb 7, 2000, 12:38:22

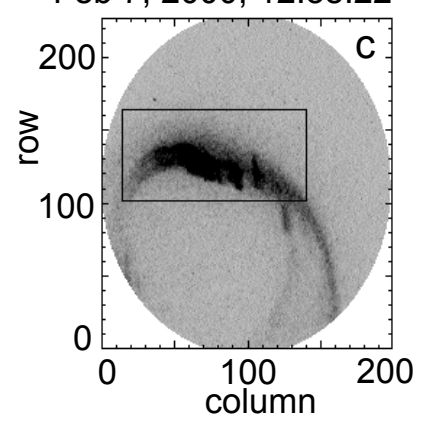

Fig. 1. (a) Auroral electrojet activity on 7 February 2000. 20-min time interval starting from the onset of intensification at $\sim 12: 27$ UT has been considered. (b), (c) Samples of Polar UVI images obtained in the time interval of interest. Fluctuations of auroral intensity in the columns of outlined regions were analyzed by the technique of logscale diagrams.

of $\mathrm{Hz}$ to $\sim 1 \mathrm{kHz}$ in the spacecraft frame is one of such manifestations. Fluctuating electric and magnetic fields in the events of BB ULF-ELF perturbations were found to exhibit power law distributions in frequency, scaling properties, nonGaussian probability density functions, and other signatures of the intermittent turbulence (e.g. Temerin, 1978; Kintner et al., 2000; Tam et al., 2005; Parkinson, 2006; Golovchanskaya and Kozelov, 2010; Golovchanskaya et al., 2011). The phenomenon of the BBELF turbulence occurs at all local times (most frequently in the prenoon sector), for any dipole tilt, in a wide variety of solar wind and geomagnetic conditions (Matsuo et al., 2003; Heppner et al., 1993). A predominant occurrence of the turbulent fields in the regions of the fieldaligned Birkeland currents was demonstrated by Golovchanskaya et al. (2006).

As shown, e.g. by Volwerk et al. (1996) and Stasiewicz et al. (1998), magnetic perturbations in the events of the BB ELF turbulence indicate strong multiscale field-aligned currents with densities of $10-100 \mu \mathrm{A} \mathrm{m}^{-2}$, whose role in such phenomena as plasma density cavitation (Stasiewicz and Potemra, 1998; Chaston et al., 2006), turbulent transport in the magnetosphere-ionosphere coupling (Lysak and Dum, 1983), formation of optical aurora at various scales (Borovsky, 1993) and others, is generally accepted.
Following the approach of Chang et al. (2004), we assume that Alfvénic activity associated with a transition to substorm expansion is accompanied by the formation of small-scale Alfvénic coherent structures of magnetostatic type. In this brief report we are searching for similarity between aurora scaling behavior at the beginning of substorm expansion and features of non-linear dynamics of Alfvénic coherent structures in the lower magnetosphere, which are simulated according to the Chang et al. (2004) scenario.

Further in Sect. 2 we show an example of statistical scaling behavior exhibited by the aurora at the early breakup stage as observed by the Polar satellite, present its quantitative characteristics and temporal evolution. In Sect. 3, by direct numerical simulation, temporal evolution of the scaling index $\alpha$ of non-linearly interacting Alfvénic coherent structures is examined. In Sect. 4, the results are summarized and discussed in the context of previous studies.

\section{Scaling behavior of the aurora at substorm early expansion as observed by Polar}

To give an example of auroral scaling behavior at the early expansion stage, we use Polar UVI images in LBH-L emission (Torr et al., 1995) with one-pixel resolution of $\sim 25 \mathrm{~km}$ obtained at the beginning of substorm activation that started on 7 February 2000, at 12:27 UT, as indicated by the AE index (Fig. 1a). The images that we treated and which samples are shown in Fig. $1 \mathrm{~b}$ displayed auroral shapes, which are common for the beginning of substorm expansion. The parts of images of $128 \times 64$ pixels size such as those delineated by rectangulars in Fig. 1b, c were selected for the scaling analysis. The data series to be analyzed were formed by joining the columns of each image. Discontinuities in auroral intensity at joint points were eliminated by adding appropriate constants. The smallest scales in the data series obtained in this manner corresponded to the structures being analyzed. This method of data preparation was tested in our previous studies (Golovchanskaya et al., 2008; Kozelov and Golovchanskaya, 2010).

Scaling features of spatial variations in auroral intensity were detected and quantified by a wavelet method, which was developed by Abry et al. (2000) and is described elsewhere (e.g. Vörös et al., 2004). Figure 2a shows the logscale diagrams (LDs) constructed by Abry et al. (2000) method for Polar UVI images obtained at different times from the time interval that started two minutes prior to the breakup onset and lasted for twenty minutes. LDs plot $y_{i}=\log _{2} \mu_{i}$, where $\mu_{i}$ is the variance of the detail wavelet coefficients $d(i, k)$ obtained by the discrete wavelet transform of the data, versus the octave (or resolution level) $i$. The discrete wavelet transform is a decomposition of the function $f(x)$ on the orthogonal basis $\left\{\psi_{i, k}\right\}_{i \in Z, k \in Z}$ composed of discrete wavelets $\psi_{i, k}$ : 
$d(i, k)=\int_{\mathbf{R}} \mathrm{d} x \psi_{i, k}(x) f(x)$,

$\int_{\mathbf{R}} \mathrm{d} x \psi_{i, k}(x) \psi_{i^{\prime}, k^{\prime}}(x)=0$ if $(i, k) \neq\left(i^{\prime}, k^{\prime}\right)$

Integers $i \in Z$ and $k \in Z$ index the scale $s=\Delta \cdot 2^{i}, \Delta$ being the smallest resolvable scale in data, and the location $x_{0}=k$. $s$. Wavelets $\left\{\psi_{i, k}\right\}_{i \in Z, k \in Z}$ are space-shifted and scale-dilated templates of a mother-wavelet $\psi_{0}$, that is,

$\psi_{i, k}(x)=\psi_{0}\left(\frac{x-x_{0}}{s}\right)$

and define a basis distributed according to a dyadic basis in the space-scale plane (Daubechies, 1988). The scaling index $\alpha_{A}$ is estimated from the slope of the weighted linear regression over the region of alignment in LDs. The uncertainty limits shown by error bars were estimated with a block bootstrap procedure (Efron, 1982; Sabatini, 1999). It should be noted that the orthogonal basis $\left\{\psi_{i, k}\right\}_{i \in Z, k \in Z}$ normalized by Eq. (2) differs from the one used in the original paper by Abry et al. (2000), therefore scaling index $\alpha_{A}$ presented here is less by 1 than that estimated with a standard LD method. For example, for fractional Brownian motion, here we should have $\alpha_{A}=2 H$, where $H$ is the Hurst exponent.

As seen from Fig. 2a, the LDs computed for individual Polar UVI images tend to reveal alignment over scales in the range from $50 \mathrm{~km}$ to $400 \mathrm{~km}$. Of course, this evidence for power law distribution of substorm aurora luminosity over scale is not new and for statistical distributions (i.e. in averaging over many events) it has been known since the study of Uritsky et al. (2002). However, for the purpose of comparison with the results of electrodynamic modeling that will be described in Sect. 3, here we focus on the temporal evolution of aurora scaling characteristics observed at the early stage of auroral breakup: the slope of the LDs undergoes a characteristic variation. Figure $2 \mathrm{c}$ displays the time evolution of scaling index $\alpha_{A}$ obtained by processing thirty one image from the time interval of interest. One can see that $\alpha_{A}$ varies from values less than one to $\sim 1.5$, increasing with breakup progress. In other words, if we interpret scaling index $\alpha_{A}$ as $\alpha_{A}=2 H$, we have a transition from a noisy anti-persistent $(H<0.5)$ signal to a smoother persistent $(H>0.5)$ one, in agreement with a similar result of Uritsky et al. (2006) obtained from the analysis of the roughness exponent of auroral surfaces during substorms. Interestingly, Kozelov and Golovchanskaya (2006) reported a similar variation of the scaling index for auroral luminosity observed during intensification of a transpolar arc under positive IMF $B_{\mathrm{Z}}$, that is, in obviously non-substorm conditions.

\section{Temporal evolution of the scaling index for non- linearly interacting field-aligned current filaments}

Following the approach of Chang et al. (2004), we assume that Alfvénic activity associated with a transition to substorm

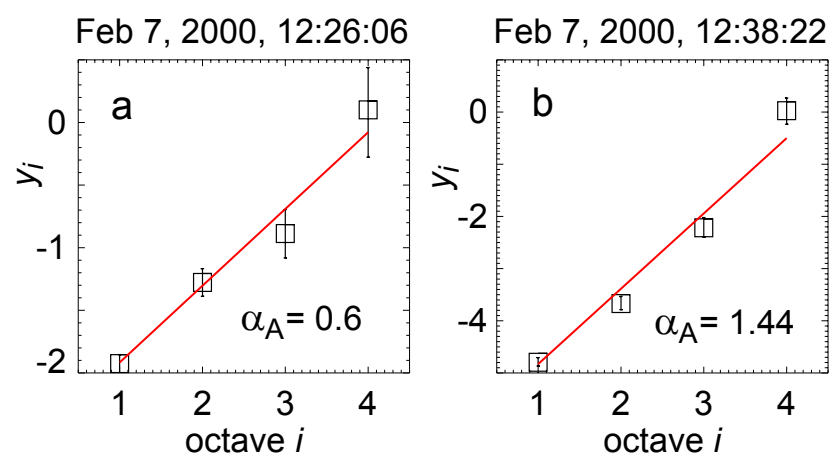

February 7, 2000

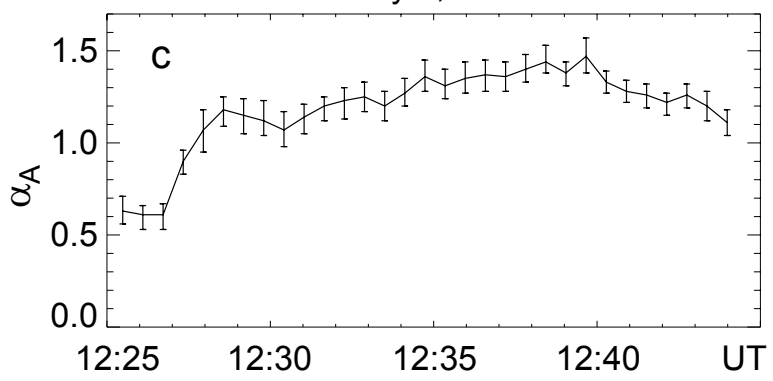

Fig. 2. (a), (b) LDs computed for individual Polar UVI images. On the abscissa axis, $i=1,2,3,4$ correspond to scales $s=50 \mathrm{~km}$, $100 \mathrm{~km}, 200 \mathrm{~km}, 400 \mathrm{~km}$. (c) Evolution of scaling index $\alpha_{A}$ with breakup progress. The uncertainty limits shown by error bars were estimated with a block bootstrap procedure for $95 \%$ confidence interval.

expansion is accompanied by the formation of Alfvénic coherent structures. As distinct from waves, these are nonpropagating fluctuations, which in the lower magnetosphere, where strong background magnetic field $\boldsymbol{B}_{0}=B_{0} \cdot \boldsymbol{e}_{\mathrm{z}}$ is present (hereinafter $\boldsymbol{e}_{\mathrm{x}}, \boldsymbol{e}_{\mathrm{y}}, \boldsymbol{e}_{\mathrm{z}}$ are unit vectors of the Cartesian coordinate system), may take the form of magnetic flux tubes (or field-aligned current filaments). Their 2-D interaction under the influence of Ampere's force is described by the equations

$\frac{\mathrm{d} \boldsymbol{x}_{m}^{( \pm)}}{\mathrm{d} t}=\boldsymbol{v}_{m}^{( \pm)}, \frac{\mathrm{d} \boldsymbol{v}_{m}^{( \pm)}}{\mathrm{d} t}=\frac{ \pm j 0}{\rho_{0}}\left[\boldsymbol{e}_{\mathrm{z}} \times \boldsymbol{B}_{m}\left(\boldsymbol{x}_{m}^{( \pm)}, t\right)\right]$

Here $\boldsymbol{x}_{m}^{( \pm)}=x_{m}^{( \pm)} \cdot \boldsymbol{e}_{\mathrm{x}}+y_{m}^{( \pm)} \cdot \boldsymbol{e}_{\mathrm{y}}$ is the coordinate and $\boldsymbol{v}_{m}^{( \pm)}=$ $v_{m, \mathrm{x}}^{( \pm)} \cdot \boldsymbol{e}_{\mathrm{x}}+v_{m, \mathrm{y}}^{( \pm)} \cdot \boldsymbol{e}_{\mathrm{y}}$ the velocity of current filament $m(m=$ $1,2, \ldots M)$ of positive $(+)$ or negative $(-)$ polarity with current density $j_{0}$, and $\boldsymbol{B}_{m}=B_{m, \mathrm{x}} \cdot \boldsymbol{e}_{\mathrm{x}}+B_{m, \mathrm{y}} \cdot \boldsymbol{e}_{\mathrm{y}}$ is the selfconsistent magnetic field acting on current filament $m$ at its location $\boldsymbol{x}_{m}=\left(x_{m}, y_{m}\right)$ at time $t$. For any particular distribution of current filaments, field-aligned current $j_{\mathrm{z}}(x, y, t)$ at the grid points can be calculated, enabling to find the magnetic flux function $\psi(x, y, t)$ as a solution of the Poisson equation

$\nabla_{\perp}^{2} \psi(x, y, t)=-\mu_{0} j_{\mathrm{Z}}(x, y, t)$ 

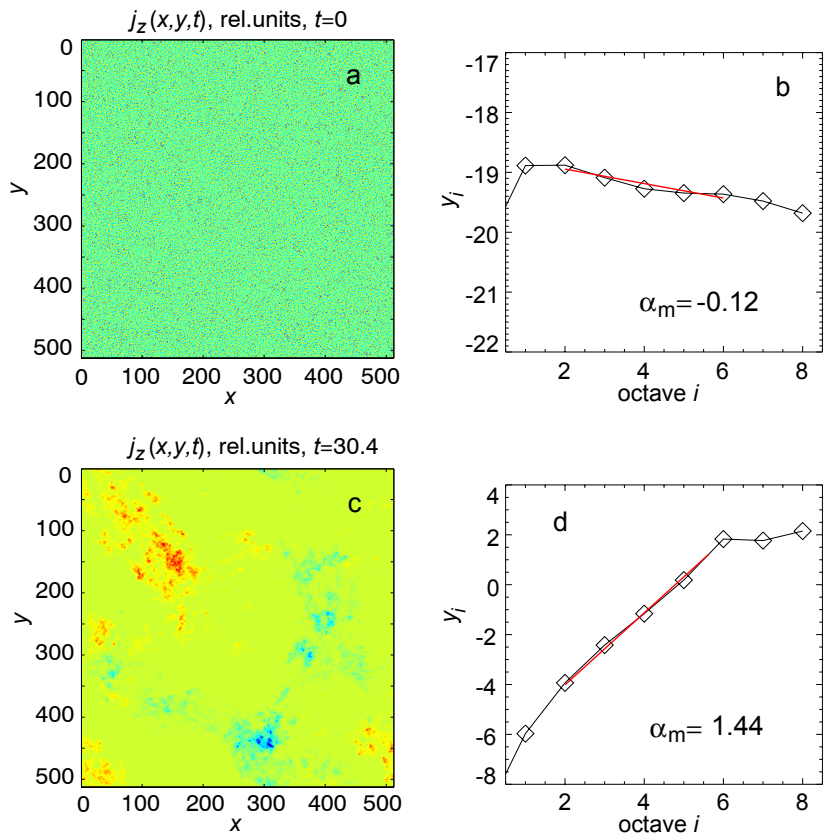

Fig. 3. Distributions in the plane perpendicular to $\boldsymbol{B}_{0}$ of simulated field-aligned currents $j_{\mathrm{Z}}$ (in relative units) produced by nonlinear interactions of Alfvénic coherent structures at (a) $t=0$ and (c) $t=30.4$, where $t$ is normalized by $t_{0}=\sqrt{\frac{\rho_{0} L_{0}}{j_{0} B_{0}}}, L_{0}$ the grid spacing, and the LDs constructed for $j_{\mathrm{Z}}$ at (b) $t=0$ and (d) $t=30.4$. Distances $x$ and $y$ are normalized by $L_{0}$.

With $\psi(x, y, t)$ known, the magnetic field at the grid points is determined as $\boldsymbol{B}(x, y, t)=\frac{\partial \psi}{\partial y} \boldsymbol{e}_{\mathrm{x}}-\frac{\partial \psi}{\partial x} \boldsymbol{e}_{\mathrm{y}}$, and $\boldsymbol{B}_{m}$ at the location of current filament $m$ is obtained by weighed averaging.

Our simulation was performed with $(513 \times 513)$ grid points. The number of current filaments $M$ of positive $(+)$ and negative $(-)$ polarities was the same and equal to $67 \times 10^{6}$. The boundary problem for Eq. (4), with periodic boundary conditions imposed, was solved with applying a method of fourth order accuracy.

At $t=0$, field-aligned current filaments of small magnitude $\left(\sim 10^{-8} \mathrm{~A} \mathrm{~m}^{-2}\right)$ are randomly distributed over the simulation region (Fig. 3a), so that the LD constructed for the field-aligned current density at $t=0$ is nearly flat (Fig. 3b), as for the white noise. At $t>0$, the non-linear motions and interactions, described by Eqs. (3) and (4), lead to clustering of small-scale current filaments of the same polarity and formation of "coarse-grained" structures of field-aligned currents (Fig. 3c) with LDs indicating power-law distributions over scales (a sample is given in Fig. 3d). The slope of the LDs grows in time, which is clearly seen from the time evolution of the scaling index $\alpha_{m}$ illustrated in Fig. 4.

Here the simulation results are shown for the case of a zero background field-aligned current, $j_{\mathrm{z} 0}=0$. When $j_{\mathrm{z} 0}$ in the form of a current-sheet is present in the problem, current filaments of the same polarity as $j_{\mathrm{z} 0}$ migrate to the sheet, producing a pattern similar to a structured auroral arc. An

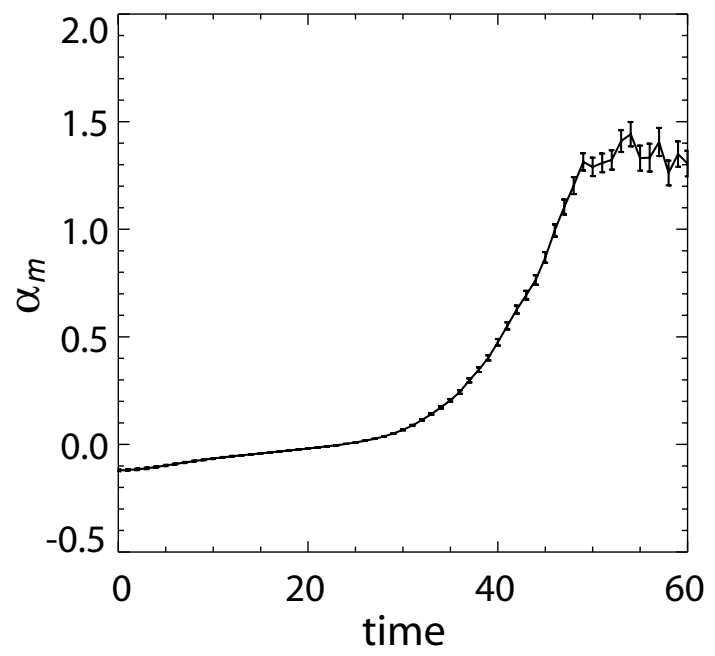

Fig. 4. Temporal evolution of scaling index $\alpha_{m}$.

example, illustrating such a pattern, was given by Chang et al. (2004).

\section{Summary}

Based on the investigation of scaling features in the substorm aurora, presented in Sect. 2 above and in the previous studies (e.g. Uritsky et al., 2006; Kozelov and Rypdal, 2007), we conclude that the involved non-linear interactions between scales indicate an inverse energy cascade. In our study this is shown from examining time evolution of scaling index $\alpha_{A}$ of auroral fluctuations at the beginning of substorm expansion by Polar imaging observations. An observed characteristic increase in the scaling index can be interpreted as due to formation of "coarse-grained" transient structures in the system near (a bit over) self-organized critical (SOC) state (Uritsky et al., 2002, 2006; Kozelov et al., 2004; Klimas et al., 2010). Numerical models of substorm dynamics based on the SOC idea have been actively discussed in numerous papers (Consolini, 1997; Uritsky et al., 2001; Milovanov et al., 2001; Kozelov and Kozelova, 2002a,b,c; Klimas et al., 2010; Vallières-Nollet et al., 2010). Unfortunately, up to now, no self-consistent theory has been proposed that would entirely describe a connection between the observed dynamics of auroral structures and the processes in the central plasma sheet from where substorms are believed to originate.

The magnetospheric substorm has been extensively studied during last decades by ground-based and satellite measurements. While there is some difference in the interpretations of details of case studies, now a common point of view is that substorm events are transients in the Earth's magnetosphere-ionosphere system that release energy loaded in the magnetospheric tail due to the coupling with the solar wind. It was shown that the tail's central plasma sheet resides in a far from equilibrium turbulent state exhibiting stochastic 
properties over a broad range of spatial and temporal scales (see e.g. surveys by Klimas et al., 2010; Stepanova et al., 2011). The magnetic reconnection in the distant tail and disruption of the cross-tail current in the near-Earth plasma sheet were suggested as the main physical mechanisms of localized energy release. Any impulsive magnetic field reconfigurations in the near-Earth magnetosphere in association with a substorm onset would launch Alfvén waves. Taking into account large amplitudes of the waves reported in numerous studies, non-linear Alfvén wave phenomena are expected. One of them is a formation of Alfvénic coherent structures. Small-scale coherent magnetic structures have been considered in the studies of Yu et al. (1981), Volokitin and Dubinin (1989), Schep et al. (1994), Pegoraro et al. (1997). By a direct numerical simulation, we investigated scaling properties of non-linearly interacting Alfvénic coherent structures of magnetostatic type, which are characterized by scaling index $\alpha_{m}$, and demonstrated that there is a general agreement in the time evolution of the indices $\alpha_{A}$ and $\alpha_{m}$.

To complete this report we note that recently Liu et al. (2011) have proposed that the interactions of the small-scale flow eddies with the magnetic field in the magnetospheric plasma sheet can self-organize into elongated current filaments and large-scale energy avalanches. A common point in the approach of Liu et al. (2011) and that of the present study is an inverse cascade driven by non-linear interactions of small-scale features. The formulation of Liu et al. (2011) is very suitable for simulation of large-scale and mesoscale processes associated with substorm activations on the nightside. On the other hand, the scaling features of auroral structures as well as the BBELF perturbations are observed in the auroral zone in both substorm and non-substorm conditions. Therefore, it is more natural to relate them to the Alfvénic activity accompanied by the formation of the coherent structures, as suggested in the present study. Alfvénic activity is especially common in association with instabilities and nonstationarities in the regions of the Birkeland currents, which can explain a predominant occurrence of the considered phenomena in such regions. We hope these effects will be verified using data of future satellite missions (Uritsky et al., 2010).

Acknowledgements. Authors thank World Data Center for Geomagnetism, Kyoto for provisional AE index data, and George Parks and Damien Chua for POLAR UVI data. This work was carried out within the Program 4 of the Presidium of the Russian Academy of Sciences (RAS) and the program "Plasma processes in the solar system" of Division of Physical Sciences of RAS.

Topical Editor I. A. Daglis thanks one anonymous referee for her/his help in evaluating this paper.

\section{References}

Abry, P., Flandrin, P., Taqqu, M. S., and Veitch, D.: Wavelets for the analysis, estimation and synthesis of scaling data, in: SelfSimilar Network Traffic and Performance Evaluation, edited by:
Park, K. and Willinger, W., p. 39, Wiley-Interscience, Hoboken, N. J., 2000.

Borovsky, J. E.: Auroral arc thicknesses as predicted by various theories, J. Geophys. Res., 98, 6101-6138, 1993.

Chang, T., Tam, S. W. Y., and Wu, C.: Complexity induced anisotropic bimodal intermittent turbulence in space plasmas, Phys. Plasma, 11(4), 1287-1299, 2004.

Chaston, C. C., Genot, V., Bonnell, J. W., Carlson, C. W., McFadden, J. P., Ergun, R. E., Strangeway, R. J., Lund, E. J., and Hwang, K. J.: Ionospheric erosion by Alfvén waves, J. Geophys. Res., 111, A03206, doi:10.1029/2005JA011367, 2006.

Consolini, G.: Sandpile cellular automata and magnetospheric dynamics, in: Proceedings of the 8th GIFCO Conference, Cosmic Physics in the Year 2000: Scientific Perspectives and New Instrumentation, edited by: Aiello, S., Iucci, N., Sironi, G., Treves, A., and Villante, U., p. 123, Soc. Ital. di Fis., Bologna, Italy, 1997.

Daubechies, I.: Orthonormal bases of compactly supported wavelets, Comm. Pure Appl. Math., 41(7), 909-996, 1988.

Efron, B.: The jackknife, the bootstrap, and other resampling plans, Society for Industrial and Applied Mathematics, Philadelphia, 1982.

Feldstein, Y. I. and Starkov, G. V.: Dynamics of auroral belt and polar geomagnetic disturbances, Planet. Space Sci., 15(4), 209229, 1967.

Golovchanskaya, I. V. and Kozelov, B. V.: On the origin of electric turbulence in the polar cap ionosphere, J. Geophys. Res., 115, A09321, doi:10.1029/2009JA014632, 2010.

Golovchanskaya, I. V., Ostapenko, A. A., and Kozelov, B. V.: Relationship between the high-latitude electric and magnetic turbulence and the Birkeland field-aligned currents, J. Geophys. Res., 111, A12301, doi:10.1029/2006JA011835, 2006.

Golovchanskaya, I. V., Kozelov, B. V., Sergienko, T. I., Brändström, U., Nilsson, H., and Sandahl, I.: Scaling behavior of auroral luminosity fluctuations observed by Auroral Large Imaging System (ALIS), J. Geophys. Res., 113, A10303, doi:10.1029/2008JA013217, 2008.

Golovchanskaya, I. V., Kozelov, B. V., Mingalev, O. V., Fedorenko, Y. V., and Melnik, M. N.: Magnetic perturbations in the events of broadband ELF turbulence observed by FAST, Geophys. Res. Lett., doi:10.1029/2011GL049003, in press, 2011.

Heppner, J. P., Liebrecht, M. C., Maynard, N. C., and Pfaff, R. F.: High-latitude distribution of plasma waves and spatial irregularities from DE2 alternating current electric field observations, J. Geophys. Res., 98(A2), 1629-1652, 1993.

Kintner, P. M., Franz, J., Schuck, P., and Klatt, E.: Interferometric coherency determination of wavelength or what are broadband ELF waves?, J. Geophys. Res., 105(A9), 21237-21250, 2000.

Klimas, A., Uritsky, V., and Donovan, E.: Multiscale auroral emission statistics as evidence of turbulent reconnection in Earth's midtail plasma sheet, J. Geophys. Res., 115, A06202, doi:10.1029/2009JA014995, 2010.

Kozelov, B. V.: Fractal approach to description of the auroral structure, Ann. Geophys., 21, 2011-2023, doi:10.5194/angeo21-2011-2003, 2003.

Kozelov, B. V. and Golovchanskaya, I. V.: Scaling of electric field fluctuations associated with the aurora during northward IMF, Geophys. Res. Lett., 33, L20109, doi:10.1029/2006GL027798, 2006.

Kozelov, B. V. and Golovchanskaya, I. V.: Derivation of 
aurora scaling parameters from ground-based imaging observations: Numerical tests, J. Geophys. Res., 115, A02204, doi:10.1029/2009JA014484, 2010.

Kozelov, B. V. and Kozelova, T. V.: Spontaneous and stimulated events in the self-organizing system and their similarity to magnetospheric mubstorms, Geomagnetism Aeronomy, 42(1), 5561, 2002a.

Kozelov, B. V. and Kozelova, T. V.: Sandpile model analogy of the magnetosphere-ionosphere substorm activity, Adv. Space Res., 30/7, 1667-1670, 2002b.

Kozelov, B. V. and Kozelova, T. V.: Positive feedback in the self-organizing model as an analogy for the magnetosphereionosphere coupling during a substorm, Geomagnetism Aeronomy, 42(4), 437-444, 2002c.

Kozelov, B. V. and Rypdal, K.: Spatial scaling of optical fluctuations during substorm-time aurora, Ann. Geophys., 25, 915-927, doi:10.5194/angeo-25-915-2007, 2007.

Kozelov, B. V., Uritsky, V. M., and Klimas, A. J.: Power law probability distributions of multiscale auroral dynamics from ground-based TV observations, Geophys. Res. Lett., 31, L20804, doi:10.1029/2004GL020962, 2004.

Liu, W. W., Morales, L. F., Uritsky, V. M., and Charbonneau, P.: Formation and disruption of current filaments in a flowdriven turbulent magnetosphere, J. Geophys. Res., 116, A03213, doi:10.1029/2010JA016020, 2011.

Lysak, R. L. and Dum, C. T.: Dynamics of magnetosphereionosphere coupling including turbulent transport, J. Geophys. Res., 88, 365-380, 1983.

Matsuo, T., Richmond, A. D., and Hensel, K.: High-latitude ionospheric electric field variability and electric potential derived from DE-2 plasma drift measurements: Dependence on IMF and dipole tilt, J. Geophys. Res., 108(A1), 1005, doi:10.1029/2002JA009429, 2003.

Milovanov, A. V., Zelenyi, L. M., Zimbardo, G., and Veltri, P.: Self-organized branching of magnetotail current systems near the percolation threshold, J. Geophys. Res., 106(A4), 6291-6307, doi:10.1029/1999JA000446, 2001.

Oguti, T.: Metamorphoses of aurora, Memoirs of national institute of polar research, Series A12, 1-101, 1975.

Parkinson, M. L.: Dynamical critical scaling of electric field fluctuations in the greater cusp and magnetotail implied by HF radar observations of F-region Doppler velocity, Ann. Geophys., 24, 689-705, doi:10.5194/angeo-24-689-2006, 2006.

Pegoraro, E., Kuvshinov, B. N., Rem, J., and Schep, T. J.: Electron inertia and small-scale magnetic structures in a non-uniform collisionless plasma, Adv. Space. Res., 19(12), 1823-1826, 1997.

Sabatini, A. M.: Wavelet-based estimation of $1 / f$-type signal parameters: Confidence intervals using the bootstrap, IEEE Transactions on Signal Processing, 47(12), 3406-3409, 1999.

Schep, T. J., Pegoraro, F., and Kuvshinov, B. N.: Generalized twofluid theory of nonlinear magnetic structures, Phys. Plasmas, 1(9), 2843-2852, 1994.

Stasiewicz, K. and Potemra, T.: Multiscale current structures observed by Freja, J. Geophys. Res., 103(A3), 4315-4325, 1998.

Stasiewicz, K., Holmgren, G., and Zanetti, L.: Density depletions and current singularities observed by Freja, J. Geophys. Res., 103(A3), 4251-4260, 1998.
Stepanova, M., Pinto, V., Valdivia, J. A., and Antonova, E. E.: Spatial distribution of the eddy diffusion coefficients in the plasma sheet during quiet time and substorms from THEMIS satellite data, J. Geophys. Res., 116, A00I24, doi:10.1029/2010JA015887, 2011.

Tam, S. W. Y., Chang, T., Kintner, P. M., and Klatt, E.: Intermittency analyses on the SIERRA measurements of the electric field fluctuations in the auroral zone, Geophys. Res. Lett., 32, L05109, doi:10.1029/2004GL021445, 2005.

Temerin, M.: The polarization, frequency, and wavelengths of highlatitude turbulence, J. Geophys. Res., 83(A6), 2609-2616, 1978.

Torr, M. R., Torr, D. G., Zukic, M., Johnson, R. B., Ajello, J., Banks, P., Clark, K., Cole, K., Keffer, C., Parks, G., Tsurutani, B., and Spann, J.: A far ultraviolet imager for the international solar-terrestrial physics mission, Space Science Rev., 71(1-4), 329-383, 1995.

Trondsen, T. S. and Cogger, L. L.: A survey of small-scale spatially periodic distortions of auroral forms, J. Geophys. Res., 103, 9405-9415, 1998.

Uritsky, V., Pudovkin, M. I., and Steen, A.: Geomagnetic substorms as perturbed self-organized critical dynamics of the magnetosphere, J. Atmos. Sol. Terr. Phys., 63(13), 1415-1424, 2001.

Uritsky, V. M., Klimas A. J., Vassiliadis D., Chua D., and Parks, G.: Scale-free statistics of spatiotemporal auroral emissions as depicted by POLAR UVI images: Dynamic magnetosphere is an avalanching system, J. Geophys. Res., 107, 1426, doi:10.1029/2001JA000281, 2002.

Uritsky, V. M., Klimas, A. J., and Vassiliadis, D.: Analysis and prediction of high-latitude geomagnetic disturbances based on a self-organized criticality framework, Adv. Space Res., 37, 539546, 2006.

Uritsky, V. M., Donovan, E., Trondsen, T., Pineau, D., and Kozelov, B. V.: Data-derived spatiotemporal resolution constraints for global auroral imagers, J. Geophys. Res., 115, A09205, doi:10.1029/2010JA015365, 2010.

Vallières-Nollet, M.-A., Charbonneau, P., Uritsky, V., Donovan, E., and Liu, W.: Dual scaling for self-organized critical models of the magnetosphere, J. Geophys. Res., 115, A12217, doi:10.1029/2010JA015641, 2010.

Volokitin, A. S. and Dubinin, E. M.: The turbulence of Alfvén waves in the polar magnetosphere of the Earth, Planet. Space Sci., 37(7), 761-765, 1989.

Volwerk, M., Louarn, P., Chust, T., Roux, A., de Feraudy, H., and Holback, B.: Solitary kinetic Alfvén waves: A study of the Poynting flux, J. Geophys. Res., 101(A6), 13335-13343, 1996.

Vörös, Z., Baumjohann, W., Nakamura, R., Volwerk, M., Runov, A., Zhang, T. L., Eichelberger, H. U., Treumann, R., Georgescu, E., Balogh, A., Klecker, B., and Réme, H.: Magnetic turbulence in the plasma sheet, J. Geophys. Res., 109, A11215, doi:10.1029/2004JA010404, 2004.

Yu, M. Y., Shukla, P. K., and Spatchek, K. A.: Alfvén wave excitation of magnetostatic modes, Phys. Fluids, 24, 1799-1801, 1981. 